\title{
Continued Advances in Supervised Autonomy User Interface Design for METERON SUPVIS Justin
}

\author{
Peter Schmaus \\ German Aerospace Center (DLR) \\ Wessling, Germany \\ Peter.Schmaus@dlr.de \\ Benedikt Pleintinger \\ German Aerospace Center (DLR) \\ Wessling, Germany \\ Benedikt.Pleintinger@dlr.de
}

\author{
Daniel Leidner \\ German Aerospace Center (DLR) \\ Wessling, Germany \\ Daniel.Leidner@dlr.de \\ Thomas Krüger \\ European Space Agency (ESA) \\ Noordwijk, The Netherlands \\ Thomas.Krueger@esa.int
}

\author{
Ralph Bayer \\ German Aerospace Center (DLR) \\ Wessling, Germany \\ Ralph.Bayer@dlr.de \\ Neal Y. Lii \\ German Aerospace Center (DLR) \\ Wessling, Germany \\ Neal.Lii@ dlr.de
}

\begin{abstract}
The exploration of the universe remains a challenging

endeavor, constantly pushing the limits of technology. Of special interest is the investigation of the other planets of our solar system such as Mars, which has been examined by various teleoperated and (semi-) autonomous satellites and landers. But an important milestone that is needed for a deeper understanding of the planet is still missing: A crewed landing. In order to send humans to such a remote location, an infrastructure for the landing crew including an energy supply, a habitat, and a return vehicle needs to be provided on the surface of the planet. The construction and maintenance of these structures is envisioned to be done by semiautonomous robots that are commanded from orbiting spacecrafts.
\end{abstract}

The teleoperation of such ground-based robots poses high demands on the capabilities of the system including robot autonomy, orbiter-robot communication, and human-robot interface design. The METERON SUPVIS Justin space telerobotics experiment suite has been initiated by the German Aerospace Center (DLR) together with the European Space Agency (ESA) to investigate the requirements for such a system and evaluate an approach. During the experiment sessions, astronauts onboard the International Space Station (ISS) command DLR's humanoid service robot Rollin' Justin on Earth to execute complex surveillance, service, and repair tasks in a simulated Martian solar farm. The robot uses its local intelligence to support the astronaut operator upon task completion allowing a simple intuitive command interface and lowering the requirements on the communication link. This work gives an overview of the developed robotic system, communication link, and tablet computer user interface (UI). In particular the tight coupling between the autonomy system of the robot and the UI, that allows the intuitive robot commanding including action parameterization, is described in detail.

The first space-ground experiment sessions of METERON SUPVIS Justin were conducted in August 2017, and March 2018 by four astronauts in total. During the first session, three astronauts demonstrated the operational readiness of our system by commanding Rollin' Justin to perform surveillance and inspection tasks. The astronauts were even able to successfully command the robot in scenarios, which were not trained prior to their spaceflight. This was possible, because our astronaut-robot collaboration concept efficiently guides the operator towards task completion. We used this property in the second experiment session to evaluate our system in even more complex scenarios. While in the first session it was sufficient for the astronaut to select the correct commands, the operator was now required to manually parameterize some of the commands to optimize the task outcome. By that, the robot has been successfully commanded to perform complex maintenance and adjustment tasks in the simulated Martian solar farm. In this work, we evaluate the preliminary results of the space-ground experiments and discuss the feedback we received from the astronauts and its impact on future space telerobotics UI design.

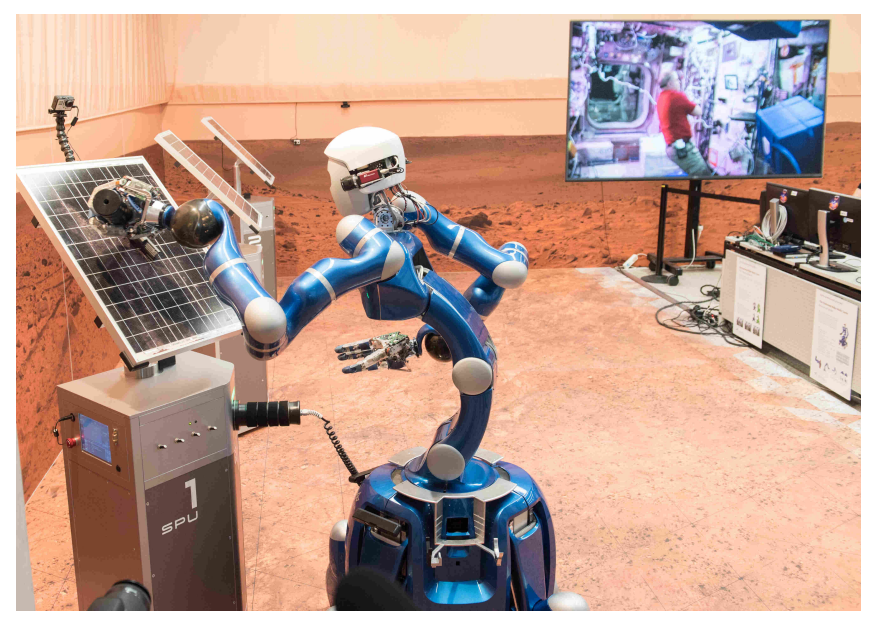

Figure 1. During the METERON SUPVIS Justin experiment session on March 2, 2018, DLR's humanoid robot Rollin' Justin was remotely operated from the

International Space Station (ISS). Live video of NASA astronaut Scott Tingle commanding the robot to clean a solar panel is displayed on the screen in the back.

\section{TABLe OF Contents}

1. INTRODUCTION..............................1

2. RELATED WORK ............................2

3. Astronaut-Robot Collaboration Concept .4

4. SPACE-GROUND EXPERIMENT ..................7

5. Conclusion ................................. 8

ACKNOWLEDGMENTS ............................9

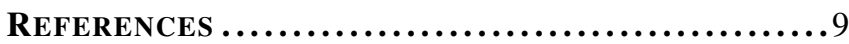

BIOGRAPHY .................................. 10

\section{INTRODUCTION}

Humans have been exploring and traveling in space for more than half a century to gather knowledge about our solar system to better understand the world we live in. For the coordination between the global space agencies, The International Space Exploration Coordination Group (ISECG) published the Global Exploration Roadmap (GER) summing up the current development and providing guidance for the focus of future space exploration missions. The GER points out, that human robot collaboration shall be an essential part of future exploration missions. As the focus of the 
agencies moves from Low Earth Orbit (LEO) missions to the crewed exploration of the surfaces of Moon and Mars, the robotic coworker supporting the astronaut crew would play an increasingly important role. Commanding these robots can be subject to a long time delay, therefore ISECG recommends in the GER to operate the robots as autonomous as possible to ensure safe and efficient robotic operations.

However, direct teleoperation of these robots is a difficult challenge. In addition to long time delay, the communication link from Earth to these robots can also be unreliable. By giving astronauts the possibility to command the robots from an orbiting spacecraft, high-bandwidth communication techniques with low delay can be employed. The low communication latency in combination with autonomous robotic operations can enable a fast-reacting human-robot team, while relieving the astronaut of laborious low-level manual commands. By handing over the task execution responsibility to a robotic coworker, the astronauts shall be able to continue with basic research or operations tasks on board the spacecraft. Ideally, the astronauts would only intervene in robotic operation when situations arise which the robot cannot handle independently. By this, the robot commanding could become a side task of the astronaut requiring an intuitive and lightweight user interface (UI).

We propose a supervised autonomy approach to realize such an UI for space service robots with local intelligence that allows the units to execute basic tasks and task sequences autonomously. By delegating the low-level planning to the robot while keeping the human operator in the loop for mission planning, the astronaut's workload is significantly reduced, as demonstrated in task-space dexterous robotic teleoperation for terrestrial application [1]. The resulting system lets the robot work for the astronaut as an intelligent coworker rather than a tool extension as depicted in Fig. 1.

Our approach is implemented in the on-going METERON SUPVIS Justin experiments, where astronauts on board the ISS command the humanoid robot Rollin' Justin on Earth. During each experiment session, different inspection and maintenance tasks were executed for the evaluation of the proposed system for real space scenarios in terms of communication link characteristics, robot intelligence, and usability of the UI. Based on the results of the first ISS-to-ground session, conducted on August 25, 2017, an updated UI version has been deployed to the ISS, and has been evaluated in the second experiment session on March 2, 2018. The changes implemented for the second experiment session together with insights on the results, are presented in this work.

The remainder of this paper is structured as follows: An overview of the related work with a focus on space telerobotics is given in Section 2. Section 3 describes the proposed astronaut-robot cooperation concept utilized for METERON SUPVIS Justin. The actual space telerobotics experiment setup, conduct, and result are detailed in Section 4. This paper concludes with an outlook on the remaining experiment session and future missions in Section 5.

\section{RELATED WORK}

The exploration of the surface of Mars is a primary target defined in the GER [2]. The first robots already pave the way for future missions by investigating the characteristics of the surface and atmosphere by scouting the environment, collecting and analyzing samples, and deploying instruments.

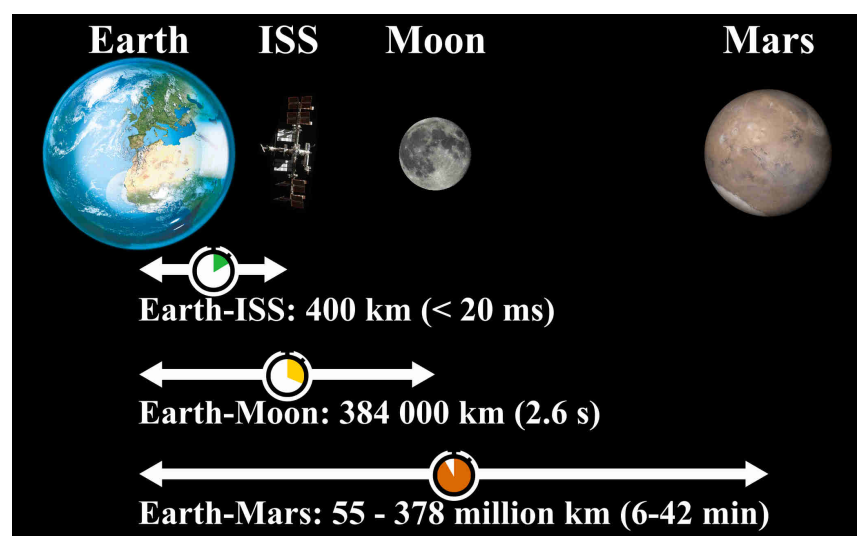

Figure 2. Overview of the distances and signal roundtrip times for communication with celestial bodies.

In addition, the focus of future robotic missions will move towards the preparation of landing sites, preparation of a return vehicle, and setup and maintenance of infrastructure needed for crewed surface exploration. Commanding these robots from Earth would be cumbersome due to the long distance between the planets, with communication delays of at least 6 minutes, up to 42 minutes in non-optimal planetary constellations, as depicted in Fig. 2. In addition to the long delay, ensuring a stable channel with as least jitter and package loss as possible also remains a challenge for deep space communication. Due to these limitations, various previous studies proposed to rather command robots on the surface from on board an orbiting spacecraft to maximize their operational effectiveness [3][4][5][6]. The proximity to the robots would allow the astronauts to use high-availability, high-bandwidth, and low-latency communication methods for robot command inaccessible for control centers on Earth.

The 2009 Avatar-EXPLORE space-telerobotics experiment by the by the Canadian Space Agency (CSA) was one of the first experiments to validate an operation concept for such crew-centered command of surface robots. During ISS expedition 20/21, a series of experiment sessions were conducted during which an astronaut had to identify thermal anomalies by commanding CSA's Mobile Robotics Test-bed (MRT) planetary rover [7] in an emulated Martian terrain to autonomously navigate towards each localized target [8]. The overall concept was to let the astronaut perform the strategic planning for task completion while leaving the execution to the rover. The non-interactive command process was similar to the command sequencing concept used for today's Mars rovers: First, the astronaut on-board the ISS received telemetry files from the robot and analyzed them in order to find the thermal anomalies. Next, the astronaut planned a robot command sequence and generated a respective command file. This file was downlinked to the robot which autonomously executed the desired commands. In three hours experiment time, one astronaut on board the ISS commanded the robot to complete six robot command sequences in total, demonstrating that the proposed operation concept is suitable for the command of semi-autonomous robots under infrequent supervision. In particular the robot's autonomy proved to be helpful to keep the robot in a safe state. This was a significant achievement as there were no possibilities for the operator to intervene in robotic operation after the command file was sent. The astronaut developed increasing trust in the robot's capabilities after becoming more familiar with the system. This resulted in more efficient utilization of the unit, and decreased data rate between robot and operator. 
From 2012 to 2014, NASA conducted the Surface Telerobotics experiment suite which simulated a future Moon exploration mission, during which astronauts on board an orbiting spacecraft had to command a surface robot on the Moon [9][10]. During the experiment sessions, NASA's K10 planetary rover was commanded by astronauts on-board the ISS to deploy a radio telescope on a simulated lunar surface at the Ames Research Center's Roverscape outdoor testbed. In contrast to the Avatar-EXPLORE experiments, NASA utilized an interactive command concept allowing the astronauts to use discrete and supervisory commands to directly command the remote robot using a high-bandwidth communication link with only up to $750 \mathrm{~ms}$ latency. During the three experiment sessions of 3.5 hours each, different astronauts commanded the robot to simulate a complete radio telescope deployment scenario consisting of three phases: (1) The first astronaut surveyed the robot's environment to identify a suitable target location for setting up the telescope. (2) The second astronaut commanded the robot to deploy the radio telescope at the previously specified target location. (3) The third astronaut inspected the telescope to verify the successful deployment. The Surface Telerobotics experiments demonstrated for the first time, the use of an autonomous robot to perform the complex procedure of setting up planetary infrastructure that is needed for future space exploration missions. The astronauts valued the autonomous functions of the robot, such as hazard detection and safeguarding, which helped to maximize robot utilization and reduced the cognitive workload of the operator while keeping the robot in a safe state. The use of interactive $3 \mathrm{D}$ visualizations of the robot in the GUI further reduced the cognitive workload and increased the situational awareness of the astronaut. With this experiment, NASA demonstrated the effectiveness of supervisory commanding of autonomous robots with interactive monitoring of the command execution for a complex construction scenario [11].

In the 2015-2017 Kontur-2 experiment suite, DLR and Roskosmos investigated the use of haptic feedback for telepresent robot commanding from a microgravity environment [12][13][14]. During the experiments, different heterogeneous robots, including a 2-Degree of Freedom (DOF) haptics evaluation robot, a rover, and a humanoid robot, were commanded by various cosmonauts on board the ISS. The cosmonauts used a 2-DOF force-feedback joystick to directly command the surface robots and feel the interaction forces as the robots made physical contact with their environment. To realize real telepresent immersion, a communication link with less than $30 \mathrm{~ms}$ latency was used, which made it possible to operate the robot to execute delicate tasks. A drawback of this fast communication link was the limitation of the experiment time to 8 minutes due to the direct line-of-sight S-Band data transmission. During various ISS-to-ground telerobotic sessions, it was demonstrated that the use of telepresent forcefeedback robot command would be an efficient approach for operating robots in prior unknown situations. The use of haptic feedback provided by the 2-DOF joystick allowed the astronauts to successfully perform tasks in which physical interaction of the robot with unknown objects of its environment was required. Even though a deterioration of the sensorimotor performance of the astronauts in microgravity was observed, the performance of the astronauts was sufficient to solve the experiment tasks and could even be compensated by providing haptic cues [15]. An important limitation of such a system is the requirement of a stable communication link with a latency below $1 \mathrm{~s}$ and minimal jitter [16].

The Multi-Purpose End-To-End Robotic Operation Network (METERON) project was initiated by ESA together with
DLR, NASA, and Roskosmos [17] with the aim to investigate effective telerobotic technologies for future space missions. As a part of METERON, the Haptics experiment suite focused on the investigation of human perception of hapticfeedback in microgravity [18][19]. In contrast to the Kontur-2 experiments, a force-feedback joystick with a single DOF was deployed for the METERON Haptics experiments. The joystick was up-massed to the ISS in 2014 together with a tablet computer and a vest that allowed a body-mounted usage of the system in addition to a conventional wall-mounted setup. During the experiment sessions, various studies were conducted such as commanding a surface robot from the ISS via a communication link with a latency of about $800 \mathrm{~ms}$. The wall-mounted joystick setup was found to be well suited for robotic operation [19]. The following METERON Interact experiment used this assembly to conduct a more complex experiment scenario [20]. During the experiment, the Interact Centaur rover located at the European Space research and TEchnology Centre (ESTEC) was commanded to execute a force-feedback-teleoperated sub-millimeter pegin-hole task. While direct mapping of Cartesian motions of the operator to Cartesian motions of the robot provides an intuitive approach towards robot command, current stateof-the-art control methods limit the use of this approach to setups with a communication link with sufficient bandwidth, low jitter, and minimal latency. In addition, the operator is always required to be in charge of all of the robot movements, resulting in high cognitive load. To relieve the astronaut, commanding the robot to navigate towards the target unit was done by placing visual assistance markers in the tablet computer Human-Robot-Interface (HRI). These markers augmented the live video feed of the robot and thus enabled intuitive commanding of the desired target robot position.

In contrast to these telepresent force-feedback approaches, the METERON SUPVIS-E and SUPVIS-M experiments of ESA shifted the focus to supervisory robot command using predefined task-level commands [21]. During the experiments, astronauts on board the ISS selected commands and monitored the telemetry of the planetary rovers transmitted via the Delay Tolerant Network (DTN), allowing for highbandwidth transmissions under variable communication delay [22]. The astronauts used laptop computers running the Meteron Operations Software (MOPS) to gain situational awareness in the remote environment, plan command sequences, issue motion- and task-level commands, and supervise the autonomous command execution by the surface robot during the ISS experiment sessions [23]. During the experiments, the system was used to command ESA's Eurobot to perform object manipulation tasks and to command ESA's Bridget rover to perform an cave exploration mission. The success of the experiments demonstrated, that task-level robot command can be highly effective in terms of lowering the cognitive and physical workload of the operator and making best use of a limited communication link for commanding a remote robot in challenging scenarios.

Although the aforementioned space-to-ground robotic teleoperation systems succeeded in commanding a specific robot in a specific scenario, none of the approaches would be well suited for direct use with different robots, scenarios or operators. In addition, these systems utilized robot-centered commands in combination with comprehensive robot telemetry displays that would require the operator to familiarize with the system and the specific robot in particular before effectively issuing commands [24]. In this work, we present a system, that overcomes these limitations by grounding the HRI design on the object-centered intelligence of the robot. 


\section{Astronaut-Robot COLlaboration CONCEPT}

In crewed spaceflight missions, autonomous robotic coworkers should take over as much work as possible to make best use of the astronauts' precious time. Ideally, the astronauts only need to intervene in robotic operations when the autonomy of the system fails. In these cases, it should be possible to command the robot as a side task during more crucial work. We propose an HRI concept that integrates the intelligence of the robot into an UI to allow for intuitive robot commanding with minimal cognitive load for the operator. An overview of the proposed concept is depicted in Fig. 3.

\section{Robot Autonomy System}

Most of today's HRIs follow a robot-centered approach where commands are defined in terms of robot actions. This approach seems to be well suited for robot movement commands but becomes more cumbersome as the complexity of the tasks increase and compliant object manipulations should be performed. In addition, high cognitive load is put on the operator as expert knowledge of the capabilities of the robotic systems is required to command such a robot to execute complex tasks. To avoid these limitations of the robot-centered approaches, we demonstrated the benefits of an object-centered organization of the robot's knowledge [25][26]. Following this approach, we store the information the robot needs to reason about and interact with its surrounding, within the context of the objects of the environment. This object-centered organization of the robot's prior knowledge allows us to use an object-oriented inheritance mechanism to reuse or specialize object properties within children objects allowing for efficient reuse of basic knowledge for different objects. The object knowledge is stored in a central Object Storage that can be shared between different robots. Using this knowledge, the robots perceive the objects in their environment and organize the obtained information about the symbolic and geometric state of the respective object instances in a World Representation.

The interaction possibilities of the robot with the objects are described by Action Templates, which generate a domain for symbolic planning and then translate the symbolic plan back to the geometric level. The symbolic state transition is described by a symbolic header specifying symbolic parameters, preconditions, and effects of the corresponding action. This information is used by the symbolic planners to find suitable object combinations. The geometric body of the Action Template grounds the symbolically planned action to the geometric level of the robot. Therefore, the geometric information regarding the symbolic parameters is resolved out of the Object Storage and used to parameterize a sequence of operations to be executed by the robot's subsystems. As each robotic system employs its own implementation of these operations, the specific way an action is executed could change depending on the physical capabilities of the specific robotic system.

The hybrid reasoning system of the robot evaluates the Action Templates of the World Representation objects' to plan the execution of feasible object interactions. Therefore, the system first resolves the symbolic header with respect to the current symbolic properties of the objects of the World Representation. The generated symbolic action schedule is then grounded to the geometric level using the respective geometric body, general object information from the Object Storage, and the current geometric object properties from the World Representation. In case no physical execution

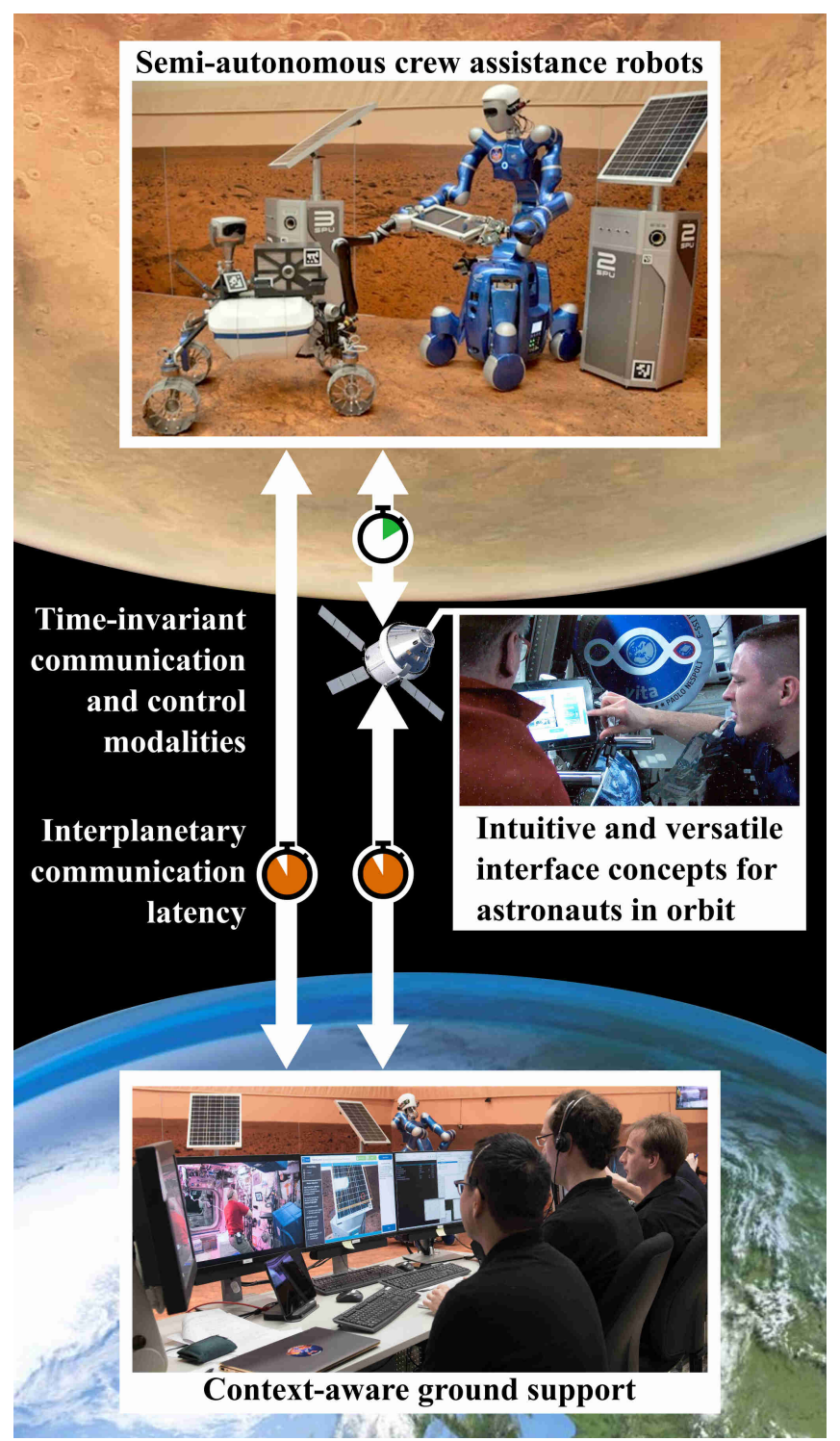

Figure 3. Schematic overview of the proposed astronaut-robot collaboration concept for commanding robots for planetary research and infrastructure maintenance from an orbiting spacecraft with support of a Earth-based Mission Control Center.

plan could be found, the hybrid reasoning system uses a backtracking mechanism to find alternative geometric and symbolic solutions to accomplish the task.

Using the hybrid reasoning system, we evaluate the Action Templates of all objects of the current World Representation to generate a set of currently feasible action options [27]. Each action option is described by a unique name and provides information about the symbolical effects of its execution, as well as a set of parameters specifying the affected objects and the action-specific execution options. The execution options define a subset of the action parameters that allow the injection of manually specified parameters into the autonomous execution planning of the robot. This gives us the possibility to tune robot actions on demand to maximize the effectiveness of the task execution. By using objectspecific information from the Object Storage in combination with output from the geometric planner, we can contextspecifically limit the parameter selection to a set of options or 
a value range, e.g. the navigation of the robot can be limited to coordinates on a predefined map. If these parameters remain unspecified, the robot would autonomously decide on the best choice depending on the task at hand.

The set of possible object actions is used for robot commanding as each option is equivalent to a high level of abstraction command the robot can autonomously execute.

\section{Context-Aware Ground Support}

Due to the flexible architecture of the Object Storage, the robot's knowledge keeps growing, resulting in more Action Templates for an increasing number of objects in the World Representation. This leads to a combinatorial explosion of possible robot commands because more and more object combinations can be used to symbolically resolve more and more Action Templates. As managing such extensive command sets can put a high cognitive load on the operator, we proposed a context-specific pruning step to remove the commands not needed to accomplish the current task [28]. Therefore, the complexity of the commands is rated according to the number of action steps that need to be performed to achieve the desired effects. Furthermore, we explicitly model the geometric relations between objects including the robot itself. Based on all this information in combination with the current object status from the World Representation, symbolic, geometric, and context-specific filters are applied to the command set in order to unclutter the information passed on to the operator.

A Mission Control Center (MCC) manages the filters in order to account for changing missions, robot capabilities, or operators. Consequently, the MCC can precisely tune the commands available to the operator for guidance towards task completion, while still keeping the operator in charge. This context-specific on-the-fly update of the command options is vital for ensuring that the operator is always provided with the best-fitting UI in the respective situation for maintaining operational readiness and quick response in critical situations.

\section{Human-Robot Interface}

As robot capability and dependability improves, commanding the robot coworker could become a low workload side task for the astronaut. This enables the astronaut to frequently, and easily switch between commanding multiple robots and other activities performed in parallel. To make the frequent switching to robot commanding possible, the HRI should be implemented for portable interface hardware, which the astronaut can carry around in the spacecraft. Tablet computers have a proving track of record for effective UI, and provide capabilities and form factor well suited for commanding autonomous robots, as we have demonstrated in previous work [27]. In addition, tablet computers are used today on board the ISS to guide the astronauts through their tasks with procedural information. As this utilization has been proven to be effective, tablet computers will likely be available on board of future crewed spacecrafts, making them a preferred device for mobile robot commanding without the need to upmass dedicated HRI hardware.

For approval to be used on board the ISS, the look-and-feel of the tablet computer Graphical User Interface (GUI) needs to comply with the ISS Display and Graphics Commonality Standard $(D G C S)$ to maintain a common look, feel, and functionality among the GUIs on board the ISS [29]. One of the concerns of the standard is the use of specific colors as some colors are reserved for specific ISS subsystems while other

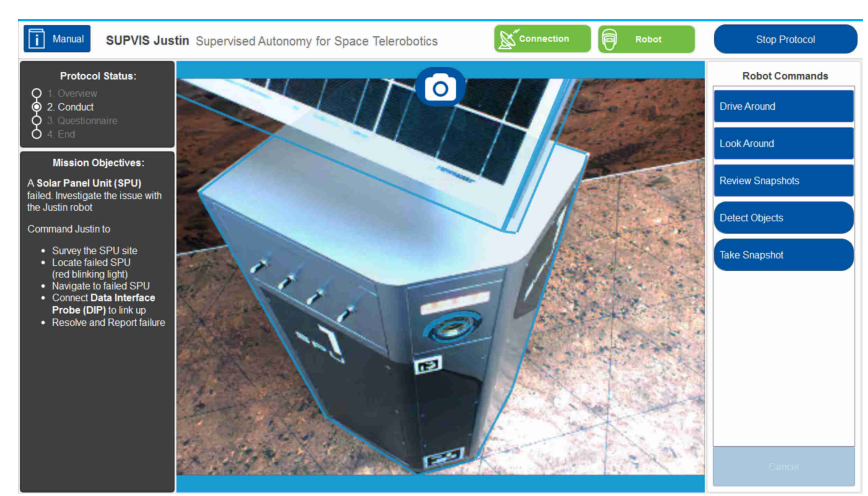

(a)

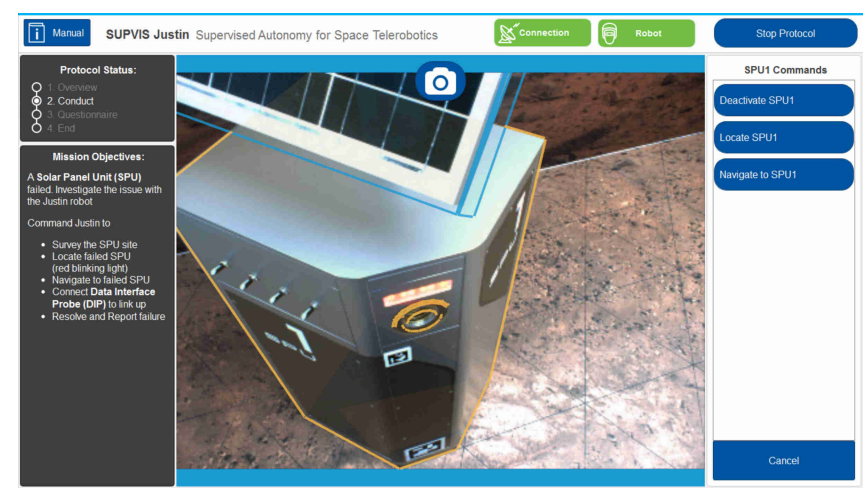

(b)

Figure 4. Screenshots of the implemented Human-Robot Interface concept for the second session of the METERON SUPVIS Justin ISS experiment: Context-specific relevant robot commands are listed at the right of the screen (a); Object-specific commands are accessed by clicking the

virtually augmented $3 \mathrm{D}$ objects in the live video and selecting a corresponding command option on the right (b)

colors are reserved for signaling specific system conditions. Therefore, we utilized a previously approved color scheme developed for the METERON Haptics experiments. In addition, we adhere to the standard terminology described in the DGCS for certain functions of the GUI, such as notification dialogues.

The use of standardized GUI elements lowers the cognitive load of the astronauts as the need for familiarization with an unknown look-and-feel concept is eliminated. This is especially helpful when using the interface for robot commanding in parallel to a different task on board the spacecraft that requires frequent switching between different interfaces. We further improve the support of the interface for these tasks switches by implementing a GUI widget showing the current mission's objectives, as well as procedural hints as shown on the left of the GUI screenshots in Fig. 4. This widget is always visible when commanding the robot to provide the astronaut with a stable entry point when returning after task switching or ensuring the mission targets. The MCC updates this display with context-specific information needed to guide the astronaut towards task completion.

The awareness of the astronaut in the remote environment of the robot is a key element to telerobotic mission success. The small screen of the tablet computer used for the HRI makes it difficult to show extensive information displays to the astronaut since the GUI elements would be too small to 
be clearly visible and a cluttered GUI would distract the astronaut from the task at hand. With our supervised autonomy approach, low-level safeguarding is performed by the robot, thus relieving the astronaut of the need to monitor lower level robot telemetry displays. As a result, we primarily rely on live video of the robot's cameras for establishing common ground between the robot and the astronaut. By augmenting the video with $3 \mathrm{D}$ renderings of the objects of the current World Representation of the robot, we inject semantic and geometric information into the video display, as visible in Fig. 4. The augmentation allows to easily infer the correctness of the robot's World Representation and the robot calibration by comparing the edges of the renderings with the real objects in the live video. We only render the outlines of the $3 \mathrm{D}$ objects in order to not occlude or distort important information.

The filtered set of commands, described in section 3, provides all context-specific reasonable robot commands. Although, the set has already been reduced by the MCC, depending on the current World Representation and task, a significant number of commands could still remain. Therefore, providing all the options to the astronaut at once can result in high workload for the astronaut and cumbersome robot commanding. We further reduce the information displayed to the astronaut to the commands of immediate interest by clustering the commands w.r.t. the affected objects. The object-related command sets can then be accessed by clicking the $3 \mathrm{D}$ objects augmenting the live video. As such, we utilize the renderings as interactive GUI elements providing access to the respective object-related commands. The point-andclick approach effectively reduces the robot commanding to (1) selecting the object that should be manipulated, and (2) selecting an object-related command from a small set of contextually meaningful commands. If no object is selected, the commands only affecting the robot itself, as e.g. navigating the environment or pointing the camera to a target location, are shown, as depiced in Fig. 4 (a). The command selected by the astronaut is autonomously planned and executed by the robot. After the robot finishes processing the command, the astronaut can correct possible execution errors by issuing respective robot commands provided by the HRI.

As described in section 3, some command parameters can be manually specified by the astronaut, such as the target position of the robot for the navigation command. To enable the astronaut to do so, selecting an parameterizeable command in the GUI does not immediately send the command to the robot but requires the astronaut to first specify the respective parameters. Depending on the type of parameters, parameter selection GUI dialogues displaying a set of sliders and/or drop-down menus are displayed to the astronaut, which allow for manual parameter selection. The GUI also provides an interface to implement command-specific parameterization GUIs that simplify the parameter selection for the astronaut. An example of such a GUI is the navigation view, that allows to specify the target position and orientation of the robot using a bird's-eye view of the environment and highlighting the currently selected navigation target. Different examples of such parameter selection GUIs are shown in Fig. 5. To make the astronaut aware of a required parameterization, these commands are displayed on rectangular buttons in contrast to buttons with rounded ends used for intermediately executing commands, as visible in Fig. 4 (a). This design choice was based on the DGCS definition of Navigation and Command buttons that is used in all ISS displays.

Commanding the robot on such a high level of abstraction, as presented in this work, allows us to lower the require-

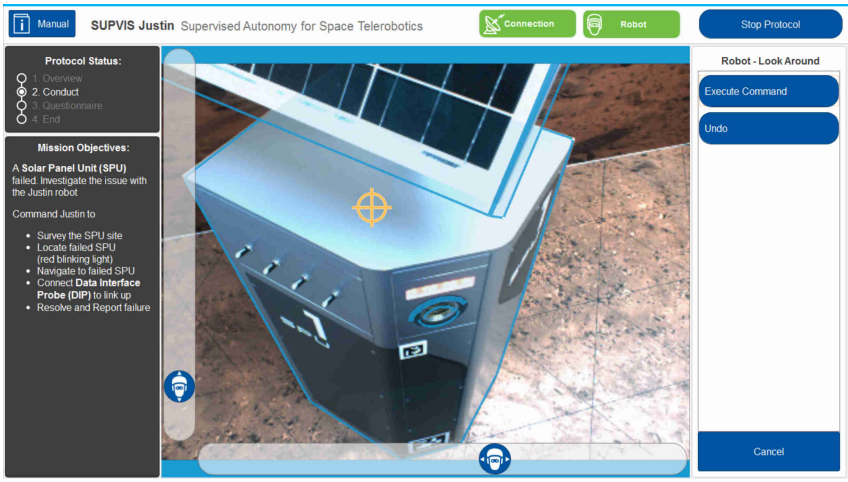

(a)

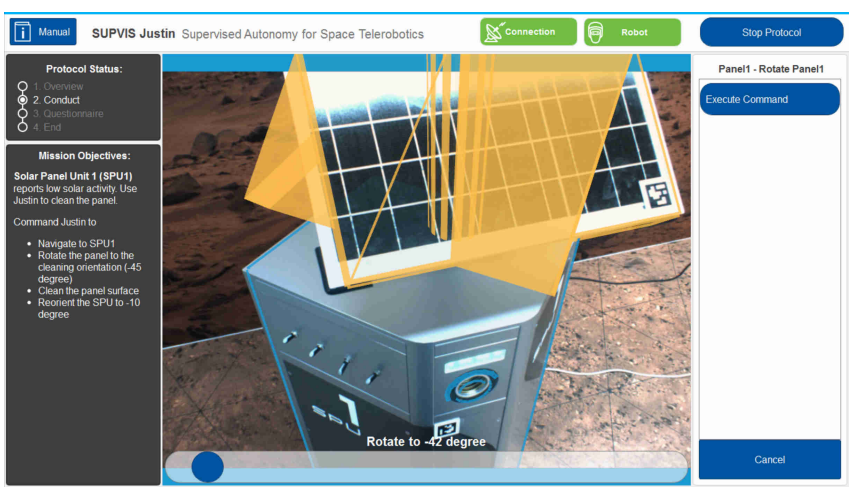

(b)

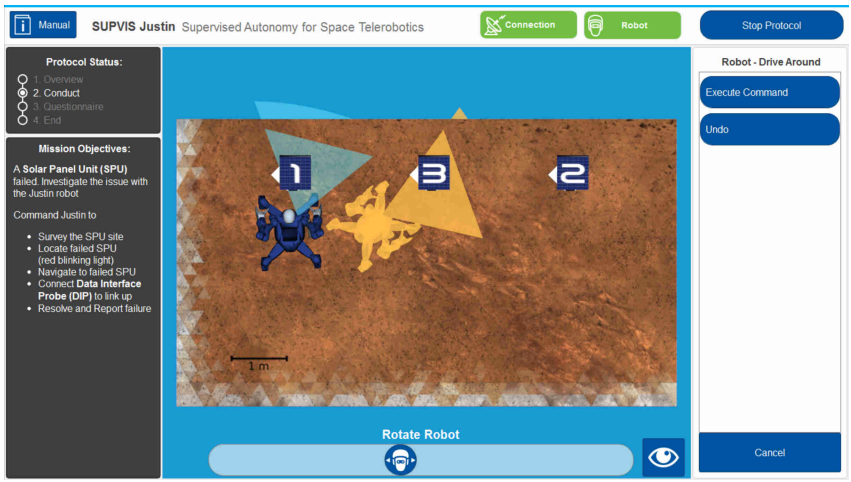

(c)

Figure 5. Screenshots of implemented parameter selection GUIs: Commanding the camera view target of the robot by positioning a crosshair (a), defining a new target orientation of the solar panel (b), and specifying a desired position and rotation of the robot in its environment (c).

ments on the communication channel compared to traditional teleoperation approaches. This is caused by the non-realtime transfer of the robot commands and responses resulting from the autonomous command execution by the robot. Using this approach, we can effectively and safely utilize communication links with frequent signal losses and high latency as the robot's autonomous capabilities always keep the system in a safe state. It is even possible to lower the live video's resolution and/or framerate to lower the required bandwidth to whatever the communication link is able to provide. This makes the system highly adaptable to situations where unreliable communication links limit the deployment of traditional teleoperation systems. 
The presented HRI concept keeps the astronaut in charge of the mission while the low-level task execution is carried out autonomously by the robotic coworker. We implemented the concept for the use on-board of the ISS and deployed the system within the METERON SUPVIS Justin space telerobotics experiment.

\section{SPACE-GROUND EXPERIMENT}

The METERON SUPVIS Justin space telerobotics experiment is a joint effort from DLR and ESA for the evaluation of the supervised autonomy HRI system presented in this work [30][31]. The experiment demonstrates the operational readiness of the system for future crewed deep space missions, where astronauts on board a spacecraft have to command semi-autonomous robotic coworkers. We use the ISS as an analogue site for the future spacecraft and set up a dexterous service robot in an analogue Martian environment at DLR, Germany, to simulate the future remote surface robot.

\section{Setup}

The SOLar farm EXperimental space robotics validation (SOLEX) environment has been constructed at DLR's Robotics and Mechatronics Center (RMC) in Oberpfaffenhofen, Germany, to allow for realistic testing and verification of the capabilities of future service robots deployed to a future extraterrestrial colony [31]. The focus of the facility lies not in simulating the martian soil or atmospheric parameters, but to provide an environment to test the robots' service capabilities in conjunction with the usability of the HRI. Therefore a fleet of three Smart Payload Units (SPUS) has been set up in the environment, which are equipped with on board computer, sensors, and battery packs for energy independence. The robot can interface with the SPUs using a set of hardware switches, a touchscreen, or a Data Interface Probe (DIP). In addition to that, the SPUs provide a set of internal and external connector sockets, allowing to equip the unit with a variety of accessories as solar panels, antennas, or computation units. In the METERON SUPVIS Justin experiment sessions, we equipped the SPUs with a solar panel which the robot can inspect, reorient, and clean. Fig. 6 shows the SOLEX environment with the SPUs as used in the ISSto-ground experiment sessions.

For our ISS-to-ground telerobotic experiments, we deployed DLR's dexterous humanoid robot Rollin' Justin, originally developed as a service robot. It consists of two DLR LWR arms with DLR Hand II endeffectors, an actuated torso with head, and an omnidirectional platform [32]. On board computers and batteries allow the robot to work independently from external data or power supply. We implemented timeinvariant whole-body control strategies that allow Rollin' Justin to precisely and compliantly interact with its environment even when commanded via an unreliable communication link [33].

The robot is connected to the ISS with the Multi-Purpose Computer and Communication (MPCC) software suite of ESA, that realizes the IP connection between the on ISS payload and on Earth systems. MPCC allows us to connect the robot to the Columbus-Control Centre $(\mathrm{Col}-\mathrm{CC})$ which is in turn directly connected to the Johnson Space Center (JSC) in Houston, Texas, USA. JSC is connected to the Huntsville Operations Support Center, where antennas provide a $\mathrm{K}_{\mathrm{u}}$-Band communication link [34] to the geostationary Tracking Data Relay Satellite System (TDRSS) [35], and finally to the ISS.

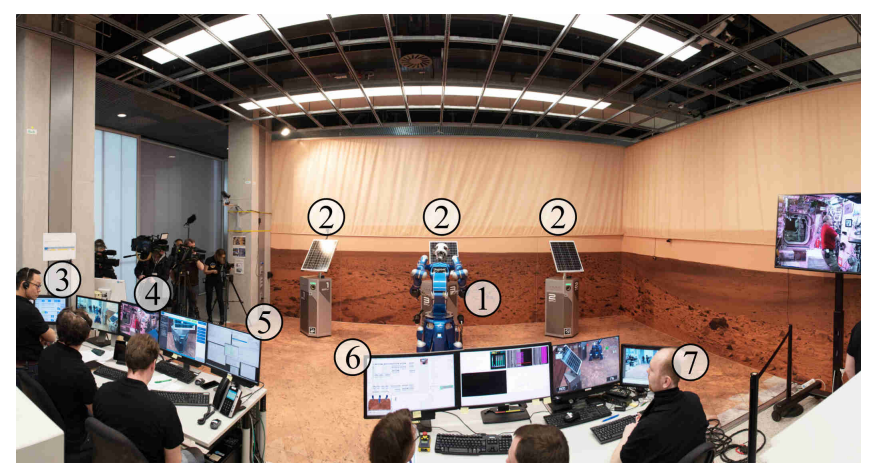

Figure 6. View of the SOLEX laboratory setup including (1) Rollin Justin, (2) Smart Payload Units, (3) PI console allowing direct voice communication with the astronaut, (4) Live ISS on board video and HRI mirror GUI, (5)

Operations monitoring station, (6) Robot console, and (7) Mission control display.

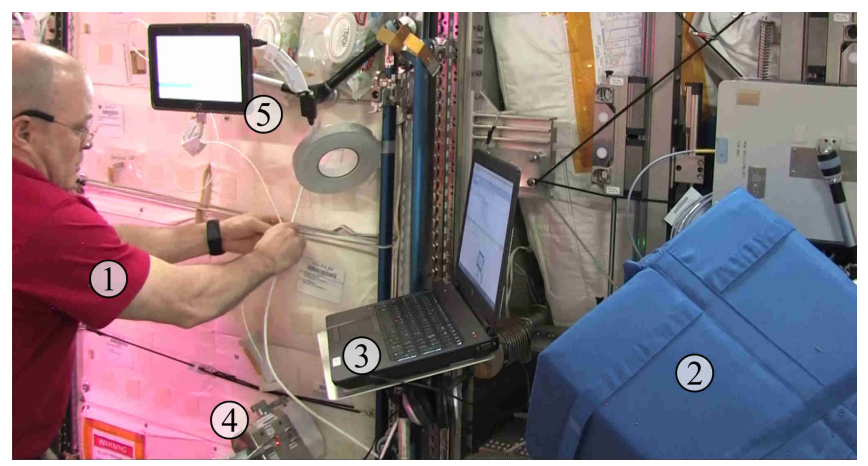

Figure 7. Setup for METERON SUPVIS Justin on board the ISS including (1) NASA astronaut Scott Tingle setting up the system, (2) the stowage box of the METERON Haptics-kit, (3) a laptop computer displaying the setup procedure, and (4) the Haptics 1-DOF joystick that is connected to (5) the tablet computer running the HRI application.

To command Rollin' Justin from the ISS, the astronaut uses the tablet computer from the METERON Haptics experiments, that has been up-massed to the ISS with ATV-5 in 2014 [18]. The tablet computer is connected to the Haptics 1-DOF force-feedback joystick with embedded computer that we use to relay the data to/from NASA's Joint Station LAN (JSL) on the ISS. The setup of the Haptics-kit inside the Columbus module of the ISS during the METERON SUPVIS Justin experiment is depicted in Fig. 7.

\section{Experiment Sequence}

The METERON SUPVIS Justin experiment incrementally evaluates the operational readiness of our system. Therefore, three ISS-to-ground sessions are conducted to test the different aspects of the HRI with an increasing level of complexity:

\section{S1) System usability validation}

In the first session, the overall usability of the HRI concept was evaluated together with an analysis of the communication channel between ISS and robot. The situational and task awareness of the astronaut were tested in scenarios involving site survey, SPU inspection including physical connection, and manipulating hardware switches of the SPU. ESA astronaut Paolo Nespoli conducted the experiment session on August 25, 2017 together with his NASA crewmates Jack Fischer and Randy Bresnik. 


\section{S2) Dexterous device adjustment and manipulation}

The second session focused on the astronaut's situational and task awareness together with the mission guidance provided by the MCC. To obtain an evaluation baseline, the robot was first commanded to execute the scenarios from the first experiment session. Afterwards, the manual command parameterization was evaluated by requesting the astronaut to optimize the efficiency of the SPU's solar panels by commanding the robot to reorient and clean the units. The ISS-to-ground teleoperation session was conducted by NASA astronaut Scott Tingle on March 2, 2018.

\section{S3) Execution of full assembly task}

According to the second session, the astronaut's performance will be related to the previous sessions by conducting the scenarios of the first session. Subsequently, a complex scenario including component installation, anomaly investigation, and component replacement will be performed to evaluate the usability of the HRI in cognitive challenging situations. The third experiment session is scheduled for August 2018.

Using the feedback of the astronauts, the MCC, and associated projected partners, we implemented updates to the GUI for the follow-up experiment sessions to improve the user experience. This allows us to dynamically adapt the HRI system to the special needs of astronauts in space. The primary changes we applied to the GUI for the second experiment session based feedback from the first astronauts was the dropping of the navigation bar on the bottom together with the integration of the different command view modes as normal robot commands. With these changes, we achieved an improved usage of the available screen space, a less cluttered GUI, and a more general approach for commanding the robot.

\section{Results}

Through our experiments, we demonstrated that we can accomplish effective teleoperation of a semi-autonomous robot by integrating the intelligence of the robot into an HRI. This way, we were able to hide the complexity of commanding a humanoid robot by providing the astronauts with an intuitive GUI. A key element of the intuitiveness of the interface was the omission of extensive telemetry displays while relying only on live video of the robots camera to generate environmental awareness. By augmenting the video with $3 \mathrm{D}$ models of the objects of the World Representation of the robot, successfully injected information about the internal status of the robot into the video stream which was used by the astronauts to decide, if the the system accuracy was sufficient, or a recalibration was needed. The astronauts assessed the situational awareness gained through the interface good suited to successfully complete the mission scenarios. In particular the overlay of the 3D objects on the live video has been assessed helpful for focusing on mission critical items of the environment. For further improve the system, they suggested to increase the field of view of the camera or allow more range of motion for pointing the camera.

The task guidance provided by our system proved to be effective, as all mission scenarios were successfully completed by the astronauts, even without providing traditional step-by-step protocol descriptions. This was accomplished by providing the astronauts with context-specific reasonable robot command sets only, which were generated using hybrid reasoning to determine the currently feasible commands in combination with mission-specific filtering by the MCC. The use of the augmented 3D objects as interactive elements to access the object-related commands proved to be effective to further reduce the complexity of the system and helped the astronaut focus on the current task. Using our HRI the astronauts were even able to command the robot in priorly unknown situations using priorly unknown commands as they were guided towards task completion by the HRI.

The use of only short command names was intended to make the GUI less cluttered and allow the astronauts to grasp the meaning of a command at a glance. Even though, we relied on standardized terminology provided by the DGCS as often as possible, and used feedback the astronauts provided after their on ground training, some command names remained ambiguous for some astronauts. One reason for that was the different previous experience of the astronauts with the command of surface robots. This is why the astronauts suggested to provide additional optional command descriptions within a future HRI to allow for on board clarification of ambiguous command names.

The supervised autonomy concept that was implemented in the proposed HRI has been demonstrated to be effective for commanding remote surface robots. By reducing the information displayed to the astronaut to a necessary minimum, we were able to implement the HRI for the use with small devices, as tablet computers. In addition, we were able to show that the astronauts were able to quickly switch from other tasks to the HRI, as was the case several times during the experiments. The portability of the interface device in combination with the quick task switching capability demonstrated the applicability of our approach for future command of robotic coworkers as a side task during other activities on board a spacecraft. The astronauts also assessed, that more complex scenarios including teams of heterogeneous robots could be commanded by the proposed HRI.

Although the astronauts had positive feedback for the low workload of the proposed HRI, all of them suggested to provide additional command modalities to command the robot on higher and lower levels of autonomy. Commands with more robot autonomy would enable the astronaut to specify mission-level commands that combine a set of actions. These commands could speed-up task completion times and further lower the cognitive load of the astronauts as the robotic coworker does more work on its own. Lower autonomy robot commands would allow the astronaut to specify basic movements of the robot as e.g. Cartesian endeffector movements or open/close hand. The low-level robot commanding, as done in classical teleoperation approaches, increases the workload of the astronaut while enabling the astronaut to handle situations the robot cannot solve autonomously. During our experiments, the astronauts suggested to provide an HRI that lets them task-specifically choose the level of autonomy for commanding the robot to be able to choose the perfect command modality to execute the current mission.

\section{Conclusion}

We successfully demonstrated the operational readiness of our supervised autonomy HRI for commanding a remote surface robot from space. The results of the first METERON SUPVIS Justin space telerobotic experiments proved the effectiveness of the UI on the ISS, and the reliability of the humanoid robot Rollin' Justin in the SOLEX environment on Earth. The concluding experiment session, scheduled for August 2018, aims to demonstrate the applicability of the system for complex tasks by confronting the astronaut with a full assembly task including component retrieval, installation, and anomaly handling, as needed for future space colony and infrastructure construction. 
As future space exploration missions will likely utilize different robot types to perform different kinds of tasks, we aim to be able to command a team of heterogeneous robots using our HRI approach. In addition, we are investigating how to adapt the granularity of the robot commands to respect special requirements of different scenarios. This includes investigating a combination of the presented supervised autonomy concept with a telepresence approach, such as used in the Kontur-2 experiment. Such a combination can enable the astronaut to solve tasks or execute motions that are completely unknown to the robot and thus cannot be executed autonomously.

Furthermore, we are currently working on transferring our research results from the space-robotics experiments to terrestrial applications within the SMiLE project investigating the use of robots to support people in need of care, such as the physically disabled and the elderly. For this use case, the HRI system developed for METERON SUPVIS Justin is modified to be able to command personal service robots in household settings by non-expert users.

\section{ACKNOWLEDGMENTS}

This work was partially supported by the Bavarian Ministry of Economic Affairs and Media, Energy and Technology, and the project SMiLE.

Implementing the ISS communication link would not have been possible without the support of the German Space Operations Center (GSOC) and the Columbus Control Centre (Col-CC). Special thanks goes to European Astronaut Training Centre (EAC) and Danish Aerospace (DAC) for their support during experiment preparation, testing, training, and execution.

\section{REFERENCES}

[1] N. Y. Lii, Z. Chen, M. A. Roa, . Maier, B. Pleintinger, and C. Borst, "Toward a Task Space Framework for Gesture Commanded Telemanipulation," in IEEE International Workshop on Robot-Human Interactive Communication (Ro-Man), 2012.

[2] International Space Exploration Coordination Group, "The Global Exploration Roadmap," www.globalspaceexploration.org, January 2018.

[3] J. Burns, D. Kring, J. Hopkins, S. Norris, T. Lazio, and J. Kasper, "A lunar L2-Farside exploration and science mission concept with the Orion Multi-Purpose Crew Vehicle and a teleoperated lander/rover," Advances in Space Research, vol. 52, pp. 306-320, 2013.

[4] W. Carey, P. Schoonejans, B. Hufenbach, K. Nergaard, F. Bosquillon de Frescheville, J. Grenouilleau, and A. Schiele, "METERON: A mission concept proposal for preparation of human-robotic exploration," in Proceedings of the IAF/AIAA Global Space Exploration Conference. International Astronautical Federation, 2012.

[5] D. Lester, K. Hodges, C. Ower, and K. Klaus, "Exploration telepresence from Earth?moon Lagrange points," in Proceedings of the IAF/AIAA Global Space Exploration Conference. International Astronautical Federation, 2012.

[6] S. Oleson, G. Landis, M. McGuire, and G. R. Schmidt, "HERRO Mission to Mars using Telerobotic Surface
Exploration from Orbit,” vol. 64, pp. 304-313, 092011.

[7] A. Gauthier, M. Pelletier, A. Salerno, P. Allard, S. Gemme, T. Lamarche, and E. Dupuis, "CSA redesign of the Mobile Robotics Test-bed (MRT)," in 2010 IEEE/ASME International Conference on Advanced Intelligent Mechatronics, 2010.

[8] E. Dupuis, P. Langlois, J. L. Bedwani, D. Gingras, A. Salerno, P. Allard, S. Gemme, R. L'Archevêque, and T. Lamarche, "The Avatar-EXPLORE experiments: Results and lessons learned," in Proceedings of the International Symposium on Artificial Intelligence, Robotics, and Automation in Space, 2010.

[9] M. Bualat, M. Deans, T. W. Fong, C. Provencher, D. Schreckenghost, and E. Smith, "ISS Crew Control of Surface Telerobots," in Proceedings of the IAF/AIAA Global Space Exploration Conference. International Astronautical Federation, 2012.

[10] J. O. Burns, B. Mellinkoff, M. Spydell, T. Fong, D. A. Kring, W. D. Pratt, T. Cichan, and C. M. Edwards, "Science on the lunar surface facilitated by low latency telerobotics from a lunar orbital platform - gateway," Acta Astronautica, 2018.

[11] M. Bualat, T. Fong, M. Allan, X. Bouyssounouse, T. Cohen, L. Flckiger, R. Gogna, L. Kobayashi, Y. Lee, S. Lee, C. Provencher, E. Smith, V. To, H. Utz, D. Wheeler, E. Pacis, D. Schreckenghost, T. Milam, D. Mittman, and R. Torres, "Surface Telerobotics: Development and Testing of a Crew Controlled Planetary Rover System," in Proceedings of the AIAA SPACE Conference, 092013.

[12] J. Artigas, R. Balachandran, C. Riecke, M. Stelzer, B. Weber, J.-H. Ryu, and A. Albu-Schaeffer, "KONTUR-2: Force-Feedback Teleoperation from the International Space Station," in IEEE International Conference on Robotics and Automation (ICRA), 2016.

[13] M. Stelzer, B. M. Steinmetz, P. Birkenkampf, J. Vogel, B. Brunner, and S. Khne, "Software Architecture and Design of the Kontur-2 Mission," in IEEE Aerospace Conference, 2017, pp. 1-17.

[14] M. Panzirsch, R. Balachandran, J. Artigas, C. Riecke, M. Ferre, and A. Albu-Schaeffer, "Haptic Intention Augmentation for Cooperative Teleoperation," in IEEE International Conference on Robotics and Automation (ICRA), 2017, pp. 5335-5341.

[15] B. Weber, S. Schätzle, C. Riecke, B. Brunner, S. Tarassenko, J. Artigas, R. Balachandran, and A. Albu-Schäffer, "Weight and Weightlessness Effects on Sensorimotor Performance During Manual Tracking," in Proceedings of the 10th International Conference on Haptics: Perception, Devices, Control, and Applications, vol. 9774. Berlin, Heidelberg: SpringerVerlag, 2016, pp. 111-121.

[16] J. Artigas and G. Hirzinger, "A brief history of DLR?s space telerobotics and force feedback teleoperation," Acta Polytechnica Hungarica, vol. 13, no. 1, pp. 239249, 2016.

[17] A. Schiele, "METERON - Validating Orbit-to-Ground Telerobotics Operations Technologies," in 11th Symposium on Advanced Space Technologies for Robotics and Automation (ASTRA), 2011.

[18] A. Schiele, M. Aiple, T. Krueger, F. van der Hulst, S. Kimmer, J. Smisek, and E. den Exter, "Haptics1: Preliminary Results from the First Stiffness JND 
Identification Experiment in Space," in Proc. of the International Conference on Human Haptic Sensing and Touch Enabled Computer Applications. Springer, 2016, pp. 13-22.

[19] A. Schiele, T. Krüger, S. Kimmer, M. Aiple, J. Rebelo, J. Smisek, E. den Exter, E. Mattheson, A. Hernandez, and F. van der Hulst, "Haptics-2 - A System for Bilateral Control Experiments from Space to Ground via Geosynchronous Satellites," in IEEE International Conference on Systems, Man, and Cybernetics (SMC). IEEE, 2016.

[20] A. Schiele, "Towards the Interact Space Experiment: Controlling an Outdoor Robot on Earth's Surface from Space," in Proc. of the 13th Symposium on Advanced Space Technologies for Robotics and Automation (ASTRA), 2015.

[21] M. Sarkarati, M. Merri, K. Nergaard, and P. Steele, "How to plug-in your rover into a space mission to moon or mars," in Automation, Robotics and Applications (ICARA), 2015 6th International Conference on. IEEE, 2015, pp. 318-324.

[22] M. Sarkarati, M. Spada, M. Merri, J. Raymaekers, and K. Nergaard, "METERON CCSDS MO Compliant Telerobotic Services Talk DTN," in AIAA SpaceOps Conference, 2014.

[23] M. Cardone, C. Laroque, M. Sarkarati, K. Nergaard, P. Steele, and S. Martin, "MOE: A System Infrastructure for Robotic Experiments," in Space Operations: Contributions from the Global Community. Springer, 2017, pp. 27-52.

[24] S. Hart, J. Kramer, S. Gee, and R. R. Burridge, "The PHARAOH Procedure Execution Architecture for Autonomous Robots or Collaborative Human-Robot Teams," in 2016 25th IEEE International Symposium on Robot and Human Interactive Communication, Aug 2016, pp. 888-895.

[25] D. Leidner, C. Borst, and G. Hirzinger, "Things Are Made for What They Are: Solving Manipulation Tasks by Using Functional Object Classes," in IEEE/RAS International Conference on Humanoid Robots (Humanoids). IEEE, 2012, pp. 429-435.

[26] D. Leidner, A. Dietrich, M. Beetz, and A. AlbuSchäffer, "Knowledge-enabled Parameterization of Whole-Body Control Strategies for Compliant Service Robots," Autonomous Robots (AURO): Special Issue on Whole-Body Control of Contacts and Dynamics for Humanoid Robots, vol. 40, no. 3, pp. 519-536, 2016.

[27] P. Birkenkampf, D. Leidner, and C. Borst, "A Knowledge-Driven Shared Autonomy Human-Robot Interface for Tablet Computers," in IEEE/RAS International Conference on Humanoid Robots (Humanoids). IEEE, 2014, pp. 152-159.

[28] D. Leidner, P. Birkenkampf, and N. Y. Lii, "Contextaware Mission Control for Astronaut-Robot Collaboration," in 14th Symposium on Advanced Space Technologies in Robotics and Automation, 2017.

[29] National Aeronautics and Space Administration, "Display and Graphics Commonality Standard," http://spaceflight.esa.int/eo/EOI/crew-in fosys/bkgnd/SSP50313RevD.pdf, SSP 50313, Accessed: 2018-09-10.

[30] N. Y. Lii, D. Leidner, P. Birkenkampf, B. Pleintinger, R. Bayer, and T. Krueger, "Toward Scalable Intuitive Telecommand of Robots for Space Deployment with
METERON SUPVIS Justin," in Proc. of the 14th Symposium on Advanced Space Technologies for Robotics and Automation (ASTRA), Leiden, The Netherlands, 2017.

[31] N. Y. Lii, A. Schiele, D. Leidner, P. Birkenkampf, R. Bayer, B. Pleintinger, A. Meissner, and B. Andreas, "Simulating an Extraterrestrial Environment for Robotic Space Exploration: the METERON SUPVISJustin Telerobotic Experiment and The Solex Proving Ground," in 13th Symposium on Advanced Space Technologies for Robotics and Automation, Noordwijk, The Netherlands, 2015.

[32] C. Borst, T. Wimbock, F. Schmidt, M. Fuchs, B. Brunner, F. Zacharias, P. R. Giordano, R. Konietschke, W. Sepp et al., "Rollin'Justin - Mobile Platform with Variable Base," in IEEE International Conference on Robotics and Automation (ICRA). IEEE, 2009.

[33] A. Dietrich, "Whole-Body Impedance Control of Wheeled Humanoid Robots," Ph.D. dissertation, Technische Universität München, 2015.

[34] A. J. Cecil, R. L. Pitts, R. N. Sparks, T. W. Wickline, and D. A. Zoller, "Extending the International Space Station Life and Operability," in AIAA SpaceOps Conference, 2012.

[35] J. Teles, M. Samii, and C. Doll, "Overview of TDRSS," Advances in Space Research, vol. 16, no. 12, pp. 67-76, 1995.

\section{BIOGRAPHY}

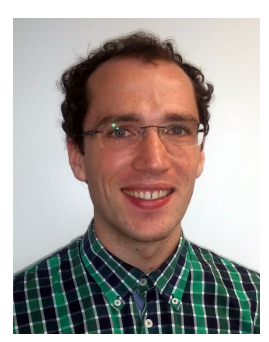

Peter Schmaus received his M.Sc. Degree in "Robotics, Cognition, Intelligence" from Technical University of $\mathrm{Mu}$ nich, Germany, in 2013. He joined the German Aerospace Center (DLR) Institute of Robotics and Mechatronics in 2011 where he was involved in the ISS-to-ground telerobotics project Kontur-2 and became Co-Investigator of the ISS-to-ground experiment suite $M E$ TERON SUPVIS Justin. His main interests lie in Shared Autonomy and effective Human-Robot Interaction.

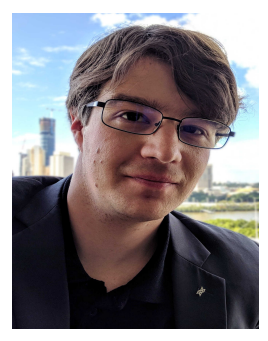

Daniel Leidner received his diploma degree in communications engineering in 2010, and his M.Sc. degree in information technology in 2011 with distinction from the Mannheim University of Applied Sciences, Mannheim, Germany. In 2017 he received the Ph.D. degree in artificial intelligence from the University of Bremen, Bremen, Germany. His dissertation was honored with the Georges Giralt PhD Award as well as the Helmholtz Doctoral Prize. He is currently leading the Rollin Justin Team and the Semantic Planning Group at the Institute of Robotics and Mechatronics, German Aerospace Center (DLR), Wessling, Germany. As Co-Investigator of the METERON SUPVIS Justin experiments, he investigates artificial intelligence in the context of astronaut-robot collaboration. 


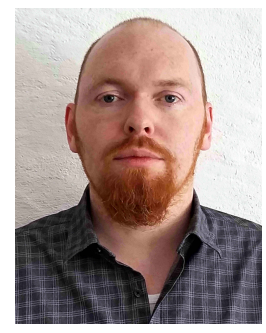

Ralph Bayer received his Master of Science in Mechanical Engineering from the Regensburg University of Applied Sciences, Germany, in 2011. He joined the German Aerospace Center (DLR) in 2010. He was a member of the development team of the five-finger dexterous DLR/HIT Hand II, as well as the space qualified force feedback joystick used for the Kontur-2 ISS-to-ground tele-manipulation experiments in 2015-2016. He currently serves as the lead thermal engineer at DLRs Institute of Robotics and Mechatronics. Mr. Bayer plays a key role in the mechanical design and integration of the ISS-to-ground telerobotic experiments, METERON SUPVIS Justin.

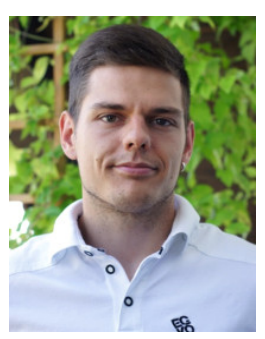

Benedikt Pleintinger received his Statecertified technical engineer in electronics in 2012 and in 2016 he received his B.Eng. in electronics from the Univerity of Applied Science Munich. He Joined the German Aerospace Center (DLR) in 2002, and the Institute of Robotics and Mechatronics in 2006 and became a member of the mechatronics department with focus on digital electronics and mechatronic system integration. He was involved in inventing of the five fingered DLR/HIT Hand II, and the mechatronic system of the humanoid walking robot TORO. As a member of the Modular Dexterous robots (MODEX) Lab he was responsible for the electrical and mechanical setup of the ISS-to-ground telerobotics experiments within METERON SUPVIS Justin.

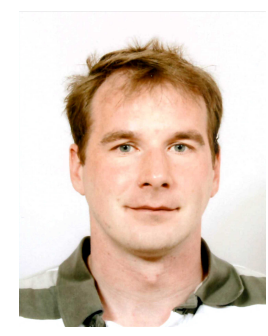

Thomas Krüger received his M. Sc. degree in electrical engineering from the University of Rostock in 2005. He extended his stay and obtained in 2008 his Ph.D. in the same field. Then he joined the European Space Agency (ESA) as a research fellow at the Telerobotics and Haptics Lab. He supported the development of controlling robots on ground from orbit in the HAPTICS and INTER-

ACT experiments. Currently he coordinates the activities of the Human-Robot Interaction Lab. In the frame of the METERON project, he supported the SUPVIS Justin experiment suite as a Co-Investigator and works now on the robotics part of ANALOG-1.

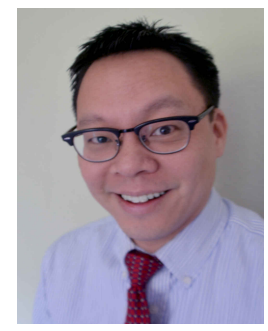

Neal Y. Lii received the Bachelor of Science in Aerospace Engineering from Purdue University, USA in 1995, Master of Science in Mechanical Engineering from Stanford University, USA in 1999, and the Ph.D. degree from University of Cambridge, UK, in 2009. He is currently the head of the Modular Dexterous robots (MODEX) Lab, as well as the domain head of Robotic Assistance, at the Institute of Robotics and Mechatronics, German Aerospace Center (DLR). In addition, he served as the Principal Investigator of the ISS-to-ground telerobotic experiments, METERON SUPVIS Justin. 